\title{
Target detectability depths of DC arrays for various models
}

\author{
Attila Novák, Sándor Szalai, László Szarka \\ Geodetic and Geophysical Research Institute of the Hungarian Academy of Sciences, \\ H-9401 Sopron POB 5, Hungary, e-mail: szalai@ggki.hu
}

\begin{abstract}
We have compared target detectability depths for six different DC geoelectric arrays. Five various $2 D$ inhomogeneity models and two noise levels ( $5 \mathrm{pc}$ and $10 \mathrm{pc}$ ) were assumed. The maximum detectability depths were determined by using the RES2DMOD software. Although the results are model-dependent (they depend both on geometry and resistivity contrast), the best results (namely: the maximum detectability depths) were obtained usually with the pole-dipole (P-DP) and the dipole axial (DP-ax) arrays. The worst results (namely: the smallest detectability depths) were obtained (with one exception) in case of the pole-pole $(P-P)$ and the Wenner- $\alpha(W-\alpha)$ arrays. The results by using the Wenner- $\beta(W-\beta)$ and dipole equatorial (Dp-eq) array groups are slightly below or above the average. Detectability depth values are comparable exclusively for the same model (partly due to the variable resistivity contrast), but in case of a certain model it can be unambiguously declared, which array is the most effective one.
\end{abstract}

Assuming $5 \mathrm{pc}$ and $10 \mathrm{pc}$ noise levels, we have carried out target detectability depth investigation for six various DC arrays and for five different subsurface inhomogeneities. Due to the fact that nowadays the largest part of DC surveys are carried out by applying multielectrode systems and Loke's RES2DINV software, we applied the corresponding forward modelling code, RES2DMOD.

In Figure 1 the definition of the detectability depth is given for a selected model. The inhomogeneity at a given depth produces an apparent resistivity anomaly image. Among the

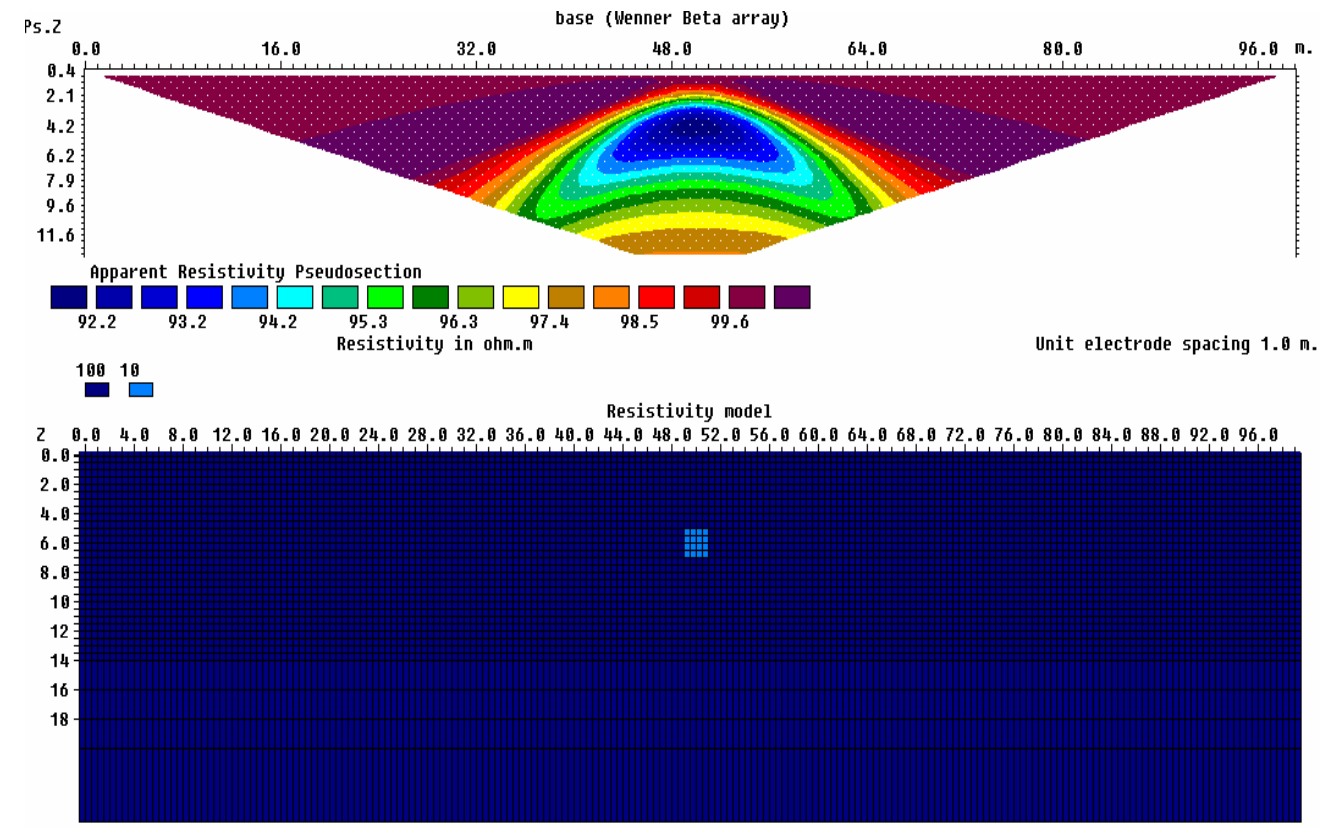

Figure 1: A resistivity model and one of its response, as an illustration of the detectability depth problem 


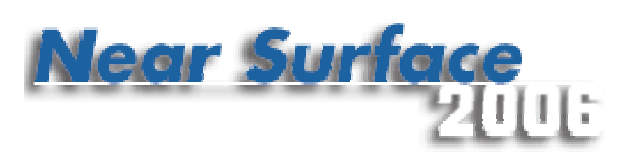

computed resistivity values we simply considered the maximum and the minimum ones ( $\rho_{\max }$ and $\rho_{\min }$ ), and we computed the ratio $100 \mid \rho_{\max }-\rho_{\min } / \rho_{1}$, where $\rho_{1}$ is the host resistivity. The model depth varied in a step of $0.5 \mathrm{~m}$ (in the depth range of $1-10 \mathrm{~m}$ ). Where the computed values became lower than 10 or 5, we fixed these depth values as target detectability depths for the given model and array, at the given noise level: $10 \mathrm{pc}$ or $5 \mathrm{pc}$.

The parameters of the forward modelling are as follows: 100 electrodes were applied, and the distance between the neighbouring electrodes was $1 \mathrm{~m}$. In case of Wenner- $\alpha$, Wenner$\beta$, pole-pole and dipole equatorial arrays 30 various electrode distances were applied; in case of pole-dipole and dipole axial arrays, the value corresponding to the dipole lengths was $\mathrm{a}=1$, and 30 different values for the distance between the dipoles were considered. The mesh data are also shown in Figure 1.

In Figures 2 the $100\left|\rho_{\max }-\rho_{\min }\right| / \rho_{l}$ values are shown for five different models and six DC arrays (two models are shown in Fig. 2a and two ones are shown in Fig. 2b).
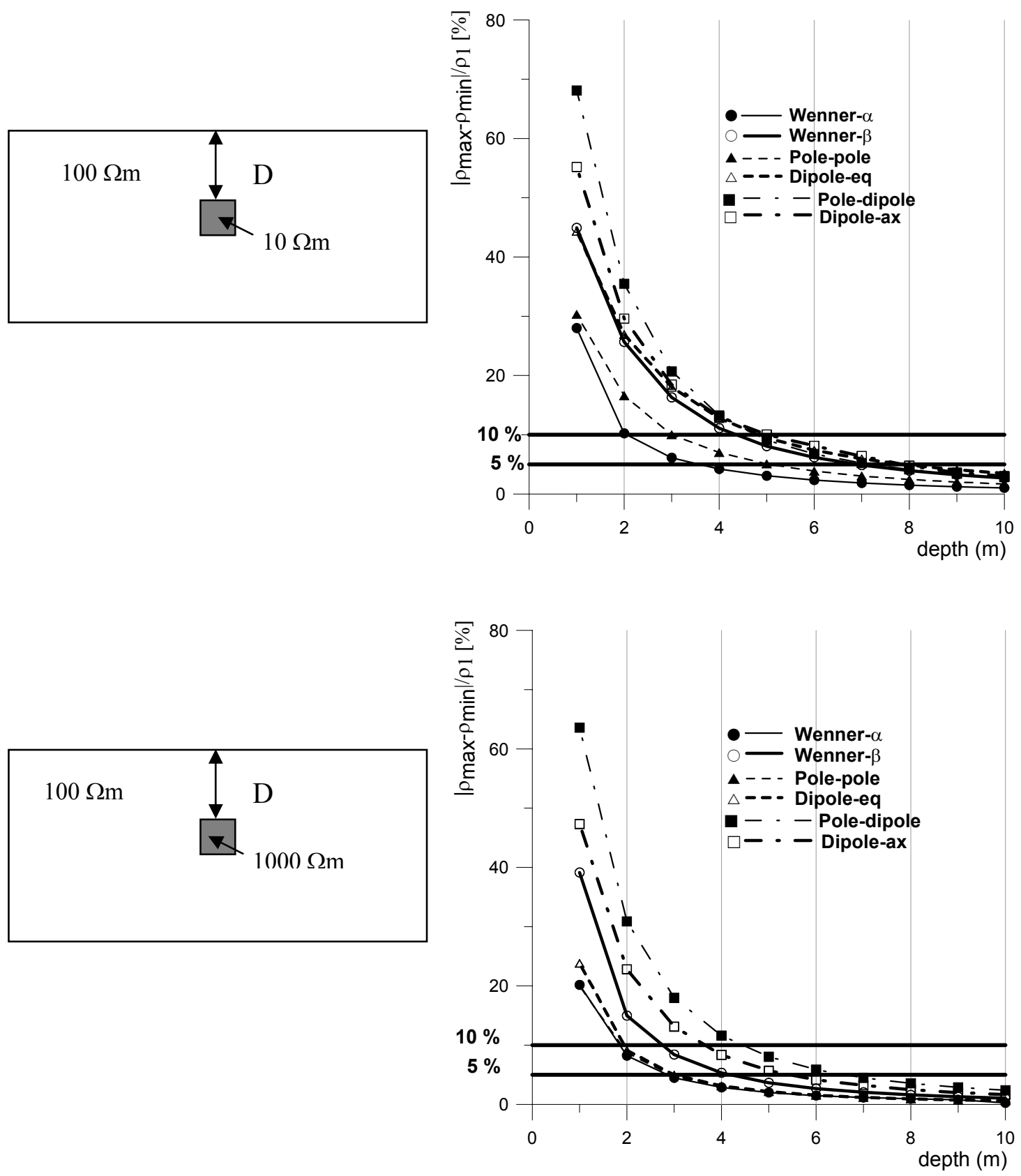

Figure 2a: Models 1, 2 and the corresponding $100\left|\rho_{\max }-\rho_{\min }\right| / \rho_{l}$ values for six DC arrays, as a function of the depth $D$ to the top of the target 

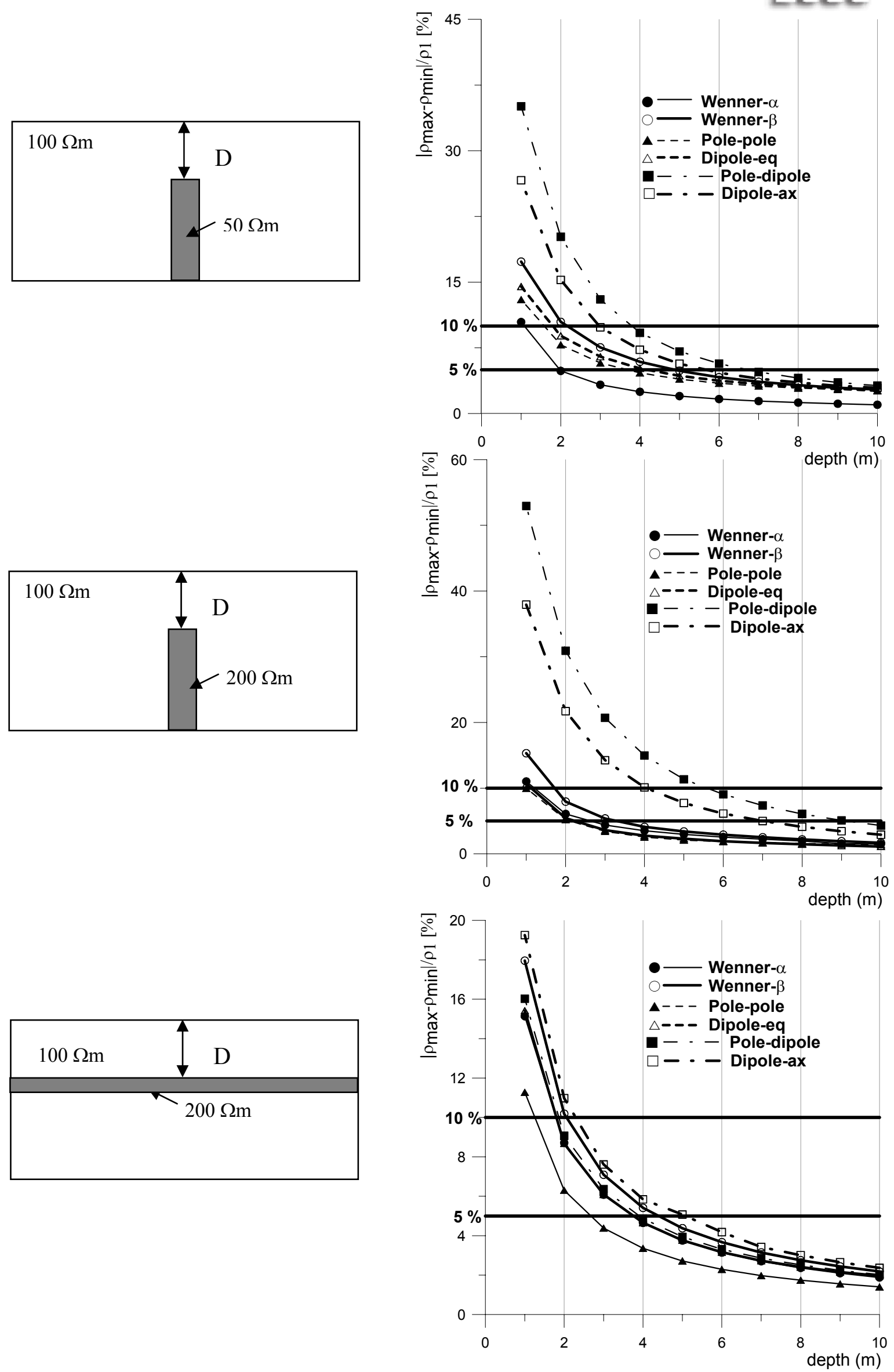

Figure 2b: Models 3, 4 and 5 and the corresponding $100\left|\rho_{\max }-\rho_{\min }\right| / \rho_{l}$ values for six DC arrays, as a function of the depth $D$ to the top of the target 
The intersections of the curves with horizontal lines of $10 \mathrm{pc}$ and $5 \mathrm{pc}$ directly provide the maximum detectability depth for the given inhomogeneity. If the inhomogeneity is at larger depth, it may by masked by various noises.

In case of the first four models (when the inhomogeneties have small lateral extension), the P-DP and DP-ax arrays proved to be the best ones. The worst results, with one single exception, were obtained by using the P-P (Figure 3 ) and $\mathrm{W}-\alpha$ arrays. In case of these narrow inhomogeneities the $\mathrm{W}-\beta$ and DP-eq arrays proved to be neither the best nor the worst arrays.
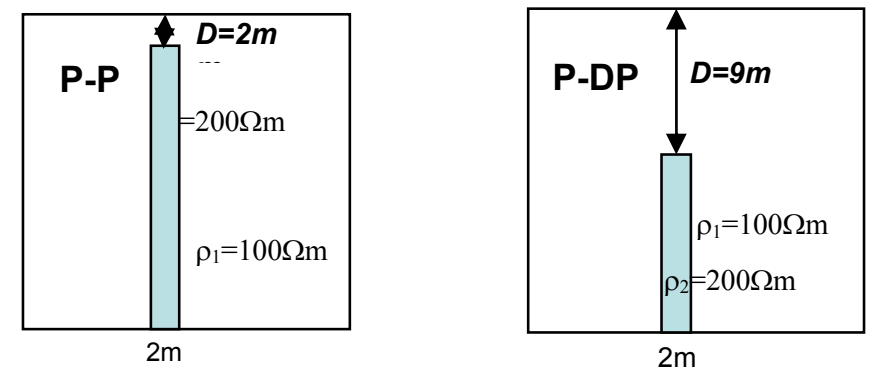

Figure 3: Detectable low-resistivity vertical slabs by using P-P and P-DP arrays

In case of model 5 (a laterally extended model), the $\beta$-type linear arrays (DP-ax and W$\beta$ ) arrays are the best ones. The worst results were obtained again by using the P-P and W- $\alpha$ arrays.

Target detectability depth values of various arrays can be compared exclusively for a given model (Table 1). In general, a low-resistivity prismatic body is detectable from larger depths than a high-resistivity one. At the same time, in case of a dyke-like geometry (when the body has a depth extension) and in case of P-DP and DP-ax arrays, high-resistivity bodies are detectable from larger depths than low-resistivity ones. (These two arrays measure the potential gradient in dip direction, while the other arrays measure either potential differences (W- $\alpha, \mathrm{W}-\beta, \mathrm{P}-\mathrm{P})$ or their derivative in strike direction (DP-eq).

\begin{tabular}{|c|c|c|c|c|c|c|c|c|c|c|}
\hline & \multicolumn{2}{|c|}{ model 1 } & \multicolumn{2}{c|}{ model 2 } & \multicolumn{2}{c|}{ model 3 } & \multicolumn{2}{c|}{ Model 4 } & \multicolumn{2}{c|}{ model 5 } \\
\hline noise & $5 \%$ & $10 \%$ & $5 \%$ & $10 \%$ & $5 \%$ & $10 \%$ & $5 \%$ & $10 \%$ & $5 \%$ & $10 \%$ \\
\hline P-DP & $\mathbf{7}$ & $\mathbf{4 . 8}$ & $\mathbf{6 . 8}$ & $\mathbf{4 . 5}$ & $\mathbf{9}$ & $\mathbf{5 . 5}$ & $\mathbf{7}$ & $\mathbf{3 . 7}$ & 4 & 1.8 \\
\hline DP-ax & $\mathbf{8}$ & $\mathbf{5 . 2}$ & $\mathbf{5 . 6}$ & $\mathbf{3 . 7}$ & $\mathbf{7}$ & $\mathbf{4}$ & $\mathbf{5 . 6}$ & $\mathbf{3}$ & $\mathbf{5}$ & $\mathbf{2 . 2}$ \\
\hline W- $\beta$ & $\mathbf{7}$ & $\mathbf{4 . 5}$ & $\mathbf{4 . 3}$ & $\mathbf{2 . 9}$ & 3 & 1.7 & $\mathbf{5}$ & 2 & $\mathbf{4 . 4}$ & $\mathbf{2 . 1}$ \\
\hline W- $\alpha$ & 3.5 & 2.2 & 3 & 1.8 & 2.5 & 1.2 & 2 & 1.1 & 3.8 & 1.7 \\
\hline DP-eq & 8 & 5 & 3 & 1.9 & 2 & 1 & 4 & 1.9 & 3.8 & 1.7 \\
\hline P-P & 5 & 3.1 & 3 & 1.8 & 2 & 1 & 3.7 & 1.8 & 2.8 & 1.3 \\
\hline
\end{tabular}

Table 1: $5 \mathrm{pc}$ and $10 \mathrm{pc}$ target detectability depth values of six DC arrays in meters for models 1-5, shown in Figure 2

The obtained target detectability depth values partly contradict to the results by Apparao et al. (1997). (In their physical modelling they only very high resistivity contrast values could be used.)

\section{Acknowledgement}

Hungarian National Research Fund, project numbers T049604 and NI 60013.

\section{References}

Apparao A., Sivarama R. S., Sarma S., 1997, Depth of detection of buried resistive targets with some electrode arrays in electrical prospecting, Geophysical Prospecting, 45, 365-375 